\title{
Impact of Exercise on Chemotherapy Tolerance and Survival in Early-Stage Breast Cancer: A Nonrandomized Controlled Trial
}

\author{
Amy A. Kirkham, PhD 1,2; Karen A. Gelmon, MD³; Cheri L. Van Patten, MSc ${ }^{3}$; Kelcey A. Bland, $\mathrm{MSc}^{4}$; Holly Wollmann, MSc ; \\ Donald C. McKenzie, MD, PhD; ; Taryne Landry, MD; and Kristin L. Campbell, BSc.PT, PhD ${ }^{5}$
}

\begin{abstract}
Background: Available preliminary evidence is conflicting on whether exercise can positively influence antineoplastic treatment tolerance and in turn improve survival. Patients and Methods: This study compared chemotherapy treatment tolerance and survival among women receiving adjuvant chemotherapy for early-stage breast cancer who participated in a single-arm trial of supervised aerobic and resistance exercise programming versus a historical cohort that did not receive structured exercise programming. Results: The exercise group (EX; $n=73$ ) and control group (CTR; $n=85$ ) participants were matched on age and treatment and balanced on medical history, cancer diagnosis, and body mass index. Attendance in the EX group was $64 \% \pm 27 \%$ of 3 offered sessions per week. For all chemotherapy agents combined, the relative risk (RR) of a chemotherapy dose reduction ( $R R, 0.78 ; 95 \% \mathrm{Cl}, 0.54-1.11)$ or delay $(\mathrm{RR}, 1.05 ; 95 \% \mathrm{Cl}, 0.62-1.80)$ did not differ between groups. However, the EX group had reduced relative and absolute risks of a dose reduction in doxorubicin by $60 \%$ and $18 \%$, respectively. For all agents combined, there were no differences between groups in risk of anemia, neutropenia, or weight gain. In the EX group, dose reductions due to neutropenia $(P=.027)$, other infections $(P=.049)$, and fatigue $(P=.037)$ were less common, whereas mucositis was more common $(P=.023)$, compared with the CTR group. The EX group had reduced relative and absolute risks of weight gain on the docetaxel + cyclophosphamide regimen by $38 \%$ and $30 \%$, respectively. After a median follow-up of 70 months (range, 54-84 months), there was no difference between the EX and CTR groups in disease-free survival events ( $n=8[11 \%]$ vs $n=9[11 \%]$, respectively; log-rank test, $P=.78$ ) or overall survival events $(n=5[7 \%]$ vs $n=6[7 \%]$, respectively; log-rank test, $P=.974)$. Conclusions: Overall, exercise programming during adjuvant chemotherapy does not appear to impact treatment tolerance or survival in women receiving common modern regimens of adjuvant chemotherapy for early-stage breast cancer. However, exercise may provide selective benefits, depending on the treatment regimen received.
\end{abstract}

J Natl Compr Canc Netw 2020;18(12):1670-1677 doi: $10.6004 /$ jnccn.2020.7603

${ }^{1}$ University of Alberta, Edmonton, Alberta, Canada; ${ }^{2}$ Now: University of Toronto, Toronto, Canada; ${ }^{3}$ British Columbia Cancer, Vancouver, British Columbia, Canada; ${ }^{4}$ Australian Catholic University, Mary Mackillop Institute for Health Research, Melbourne, Victoria, Australia; and ${ }^{5}$ University of British Columbia, Vancouver, British Columbia, Canada.

\section{Background}

One of the most compelling questions about the role of exercise in clinical oncology is whether it can positively influence antineoplastic treatment tolerance or efficacy, thereby reducing the risk of cancer recurrence and improving survival. ${ }^{1}$ Proposed mechanisms for this positive effect on chemotherapy treatment tolerance include exercise-related amelioration of specific symptoms or toxicities that cause treatment reductions or delays, including neutropenia, fatigue, and neuropathy. ${ }^{1,2}$ In turn, receipt of the full intended chemotherapy dose is a determinant of disease-free survival (DFS) and overall survival (OS). ${ }^{3}$

There are no published exercise trials with a measure of cancer treatment tolerance, treatment efficacy, or survival outcome as the primary outcome or as secondary outcomes with sufficient statistical power. ${ }^{4}$ Two large randomized controlled trials (RCTs) have reported exploratory findings indicating that supervised exercise training concurrent to chemotherapy for early-stage breast cancer improved chemotherapy completion rates relative to usual care. ${ }^{2,5}$ In addition, exploratory long-term follow-up of 2 large RCTs showed that exercise was associated with a (non-statistically significant) $32 \%$ to $34 \%$ improvement in DFS. ${ }^{6,7}$ The impact of exercise on cancer recurrence and survival is currently being assessed in 4 adequately powered ongoing RCTs with sample sizes up to 962 patients. ${ }^{8-11}$ Until these data are available, use of a historical control group provides the opportunity for exploratory analyses to provide key insights into the potential of exercise to influence antineoplastic treatment tolerance or survival. ${ }^{12}$

The purpose of this study was to examine chemotherapy treatment tolerance and survival among women receiving adjuvant chemotherapy for early-stage breast cancer who participated in a single-arm intervention trial of structured aerobic and resistance exercise programming compared with a historical cohort of similar patients who did not receive structured exercise programming. A secondary purpose was to determine whether the impact 
of exercise on these outcomes differed by chemotherapy regimen.

\section{Patients and Methods}

\section{Design and Participants}

The study design was an exploratory analysis of a nonrandomized controlled trial using a single-arm intervention and a historical control. Patients in both groups were adult women diagnosed with stage I-IIIA breast cancer and received adjuvant chemotherapy treatment at the Vancouver center of British Columbia (BC) Cancer in Canada. The individuals in the intervention group were enrolled in the Nutrition and Exercise During Adjuvant Treatment (NExT) trial (ClinicalTrials.gov identifier: NCT01806181), which assessed the effectiveness of a supervised exercise and healthy eating program offered as part of supportive care in a real-world setting. ${ }^{13-16}$ Patients could enroll in NExT any time between 2 weeks before the first chemotherapy treatment and completion of $>50 \%$ of planned chemotherapy. Exclusion criteria included body mass index $>40 \mathrm{~kg} / \mathrm{m}^{2}$, impaired mobility (eg, wheelchair/cane use), uncontrolled cardiovascular disease, or uncontrolled diabetes.

The historical control group was randomly selected by the Breast Cancer Outcomes Unit at BC Cancer, which was not otherwise involved in the study. The potential pool of patients received a medical oncology consultation for adjuvant therapy within the 18 months before the period of active recruitment for NExT. No structured exercise programing was available at BC Cancer during this time period, and treatment approaches were similar. Control participants were matched 2:1 to NExT participants using age at diagnosis within 2 years. After the matching process, additional exclusion criteria were applied to the control participants: (1) receipt of treatment at other BC Cancer locations that might introduce geographical differences in outcomes and/or difficulty in attaining complete medical records; (2) receipt of first chemotherapy after the NExT recruitment initiation date (ie, could have been eligible for NExT but were not referred for unknown reasons, and therefore was considered an inappropriate comparison); and (3) medical oncology consultation was to initiate hormone therapy and patient had already completed adjuvant chemotherapy before the period of interest.

The study was approved by the BC Cancer Research Ethics Board, and the women who participated in NExT provided informed consent.

\section{Intervention}

The exercise intervention has been described in detail previously. ${ }^{14,15}$ The exercise prescription was based largely on a previous efficacy trial ${ }^{17}$ and consisted of supervised, progressive, combined aerobic and resistance exercise training 3 times weekly. Aerobic exercise progressed from moderate to vigorous intensity $(50 \%-75 \%$ of age-predicted heart rate reserve) and from 20 to 30 minutes over the first 9 weeks of the program. Resistance training was progressed from 1 to 2 sets of 10 to 12 repetitions at an intensity of $50 \%$ to $75 \%$ of estimated one-repetition maximum for leg press and chest press, and a similar degree of difficulty for leg curls, calf raises, seated row, biceps curls, and triceps extensions. The intervention continued for the length of chemotherapy and radiotherapy (if received), after which the frequency of supervised sessions was reduced in a stepwise fashion (twice per week for 10 weeks, then once per week for 10 weeks). Participants also attended a single groupbased nutrition counseling session discussing symptom management, including weight management briefly.

\section{Outcomes}

The primary treatment tolerance outcome was any reduction in dose of a single chemotherapy agent from the planned dose. Secondary treatment tolerance outcomes included (1) delays in receipt of chemotherapy ( $\geq 5$ days later than planned); (2) relative dose intensity (RDI) $^{18}$; (3) receipt of $<85 \%$ of RDI; (4) prevalence of reasons for dose reduction or delay; (5) risk and severity of neutropenia or anemia (defined by NCI's CTCAE version 5.0) for $\geq 1$ treatment; and (6) risk of weight gain during chemotherapy $(\geq 0.5-\mathrm{kg}$ increase from before chemotherapy to the last available body weight measurement at the end of chemotherapy, based on the average amount of body weight variation expected for a period of 3-6 months)..$^{19}$

Exploratory survival outcomes were OS (defined as time from diagnosis to death from any cause) and DFS (defined as time from diagnosis to time of any recurrence in the ipsilateral breast or in nodal or distant sites, a contralateral breast cancer, or death from any cause).

\section{Clinical Data Extraction}

Diagnosis and treatment data were extracted from medical records in a standardized and identical manner for both groups. Body weight was extracted from chemotherapy treatment records. CBC counts from before chemotherapy and after every treatment cycle were extracted to determine prevalence of anemia and neutropenia. Cancer recurrence and mortality data were provided by the Breast Cancer Outcomes Unit current to February 20, 2019.

\section{Analysis}

Baseline characteristics were compared between groups using independent $t$ tests and chi-square tests. Relative risk (RR; with 95\% confidence intervals and number needed to treat $[\mathrm{NNT}]$ ) was used to compare categorical 
metrics of treatment tolerance, whereas independent $t$ tests or Mann-Whitney $U$ tests were used for parametric or nonparametric continuous variables, respectively. The likelihood of reasons for dose reductions and delays versus the number of planned/received cycles were compared between groups using chi-square or Fisher exact tests when cell size was $>5$ or $<5$, respectively. For anemia and neutropenia severity, a chi-square test was used to compare the number of cycles at each level between groups. The Kaplan-Meier product-limit method was used to describe survival probabilities for DFS and OS outcomes. The logrank test was used to assess differences in the (unadjusted) survival probabilities between groups.

The primary analyses were performed using an intention-to-treat (ITT) approach for the exercise group, in which all enrolled patients were included. Perprotocol analyses were also performed with inclusion of only those who attended $\geq 50 \%$ of all exercise sessions prescribed during chemotherapy. For brevity, the perprotocol analysis results are reported only when they differ from the ITT results.

Subgroup analyses were performed to determine whether the impact of exercise on treatment tolerance differed by specific chemotherapy agents. These analyses were performed using ITT only due to smaller sample sizes. The RR of a dose reduction or delay for doxorubicin, docetaxel, and paclitaxel was assessed independently. Cyclophosphamide was not assessed because it was exclusively administered concurrent to doxorubicin or docetaxel and all participants received this agent. The RR of weight gain across the entire length of treatment was assessed for the 2 main regimen types separately (ie, docetaxel + cyclophosphamide, or doxorubicin + cyclophosphamide \pm sequential paclitaxel). Comparisons of reasons for dose reductions and delays were performed for all chemotherapy regimens together.

\section{Results}

\section{Patients}

Between August 5, 2013, and October 31, 2014, 93 eligible patients were referred and 73 enrolled in the NExT exercise program (EX group) (Figure 1). Nine patients requested withdrawal during chemotherapy but were included in the ITT analyses. All patients received regimens consisting of either docetaxel + cyclophosphamide (29\%), or doxorubicin + cyclophosphamide \pm sequential paclitaxel $(71 \%)$; one-third also received trastuzumab (Table 1). During chemotherapy, adherence to the prescribed exercise frequency (3 times weekly) was

\section{NExT Group (Exercise)}

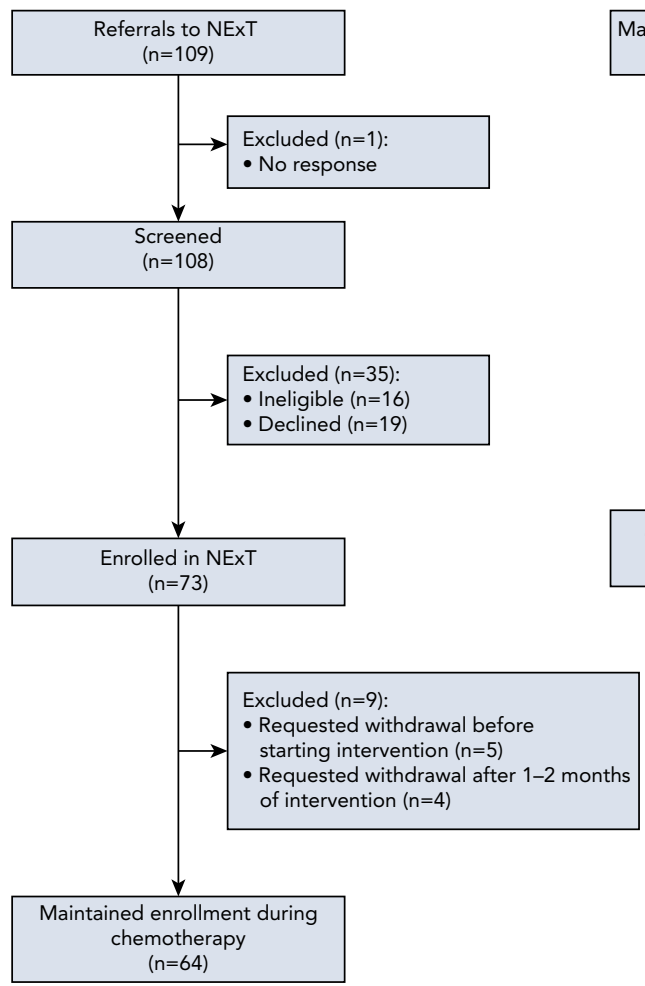

\section{Control Group}

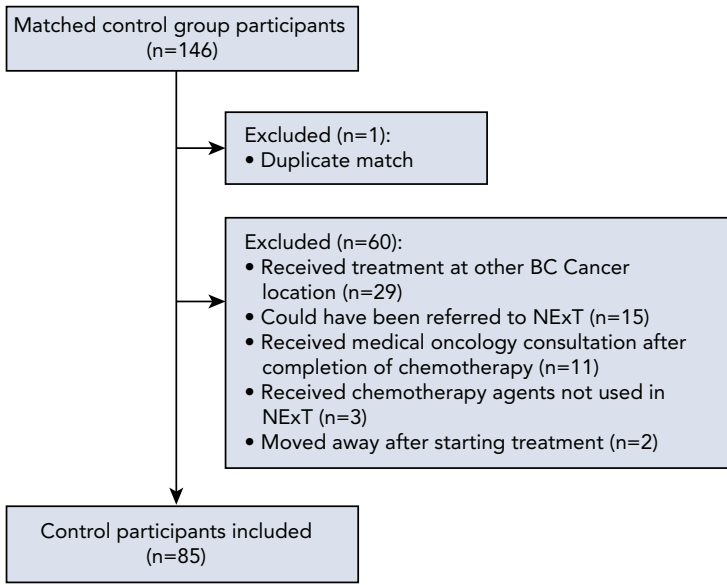

Figure 1. Flow diagrams of patient selection.

Abbreviation: NExT, Nutrition and Exercise During Adjuvant Treatment. 
$64 \% \pm 25 \%$; 49 participants (67\%) attended at least half of the scheduled sessions. Average adherence to exercise frequency did not differ between those receiving docetaxel + cyclophosphamide $(63 \% \pm 27 \%)$ versus those receiving doxorubicin + cyclophosphamide \pm paclitaxel $(58 \% \pm$ $30 \% ; P=.290$ ). Adherence to the prescribed aerobic exercise intensity and duration were $73 \% \pm 20 \%$ and $82 \% \pm$ $20 \%$, respectively. Adherence to all components of the prescribed resistance program (sets, repetitions, and weights for each exercise) was $57 \% \pm 23 \%$. Detailed exercise adherence data have been reported previously. ${ }^{15}$

The historical control participants $(n=146)$ attended a new medical oncology consult for early-stage breast cancer between February 1, 2012, and August 5, 2013. Medical records were screened for the additional eligibility criteria outlined earlier, resulting in exclusion of 57 control participants (Figure 1) and 1 control participant who was matched to 2 NExT participants and therefore was included in the control group only once. A post hoc decision, blinded to outcomes, was made to exclude 3 additional control participants who had received chemotherapy protocols containing different classes of agents not used to treat any patients enrolled in NExT (ie, fluorouracil and carboplatin) in order to remove the confounding variable of treatment regimen from our analysis. In total, outcomes were extracted for 85 control participants (CTR), with $34 \%$ receiving docetaxel + cyclophosphamide, $67 \%$ receiving doxorubicin + cyclophosphamide \pm paclitaxel, and $26 \%$ also receiving trastuzumab. The groups did not differ in the prevalence of each chemotherapy regimen for patients with stage I, II, or III cancer. The EX and CTR groups were balanced on diagnosis and treatment characteristics (Table 1).

\section{Chemotherapy Tolerance}

The RR of a reduction in chemotherapy dose did not differ between the EX and CTR groups (38\% vs $49 \%$, respectively; RR, 0.78; 95\% CI, 0.54-1.11; $P=.170$; NNT, 9) (Figure 2A). The RR of a chemotherapy delay also did not differ between the groups ( $26 \%$ vs $25 \%$, respectively; RR, 1.05; 95\% CI, 0.62-1.80; $P=.845$; NNT, 76). RDI (median [min, max], 96 [23, 103] vs 95 [17, 100]; $P=.209$ ) and the RR of a chemotherapy dose reduction to $<85 \%$ of RDI (16\% vs $24 \%$; RR, 0.70 ; 95\% CI, 0.37-1.33; $P=.275$; NNT, 14) did not differ between the EX and CTR groups, respectively. However, using per-protocol analysis, a trend was seen toward a higher RDI $(97 \%[74,103]$ vs $95 \%$ [17, 100 ], respectively; $P=.064)$ and a reduced risk of a dose reduction to $<85 \%$ RDI ( $12 \%$ vs $24 \%$, respectively; RR, 0.43; 95\% CI, 0.17-1.08; $P=.073$; NNT, 8), in favor of EX.

The RR of experiencing neutropenia for at least one chemotherapy cycle did not differ between groups (both 59\%; RR, 1.00; 95\% CI, 0.77-1.30; $P=.987$; NNT, 757; Figure $3 \mathrm{~A})$. The absolute or relative (percentage of total)

\begin{tabular}{|c|c|c|c|}
\hline Variable & $\begin{array}{c}\text { NExT (Exercise) } \\
\text { Group } \\
\text { n (\%) }\end{array}$ & $\begin{array}{l}\text { Control } \\
\text { Group } \\
\text { n (\%) }\end{array}$ & $\begin{array}{c}P \\
\text { Value }\end{array}$ \\
\hline Patients, N & 73 & 85 & \\
\hline Age, y & $50.8 \pm 10.6$ & $50.2 \pm 10.4$ & .670 \\
\hline Body mass index, $\mathrm{kg} / \mathrm{m}^{2}$ & $26.1 \pm 5.9$ & $25.7 \pm 5.1$ & .580 \\
\hline Menopausal status & & & .205 \\
\hline Premenopause & 40 (55\%) & 55 (65\%) & \\
\hline Postmenopause & $33(45 \%)$ & $30(35 \%)$ & \\
\hline \multicolumn{4}{|l|}{ Comorbidity } \\
\hline Hypertension & $12(16 \%)$ & $10(11 \%)$ & .398 \\
\hline Mental illness & $11(15 \%)$ & $8(9 \%)$ & .276 \\
\hline Arthritis/Osteoporosis & $8(11 \%)$ & $6(7 \%)$ & .390 \\
\hline Asthma/Lung disease & $7(10 \%)$ & $3(3 \%)$ & .189 \\
\hline $\begin{array}{l}\text { Cardiovascular disease/ } \\
\text { arrhythmias }\end{array}$ & $5(7 \%)$ & $4(5 \%)$ & .734 \\
\hline Metabolic disease & $3(4 \%)$ & $5(6 \%)$ & .726 \\
\hline Thyroid disorder & $3(4 \%)$ & $5(6 \%)$ & .726 \\
\hline Tumor stage & & & .208 \\
\hline $\mathrm{IA} / \mathrm{B}$ & $17(23 \%)$ & $29(34 \%)$ & \\
\hline$\| A / B$ & 47 (64\%) & $43(51 \%)$ & \\
\hline IIIA & $9(12 \%)$ & $13(15 \%)$ & \\
\hline Tumor size & & & .950 \\
\hline $\mathrm{T} 1$ & $30(41 \%)$ & 37 (44\%) & \\
\hline $\mathrm{T} 2$ & 37 (51\%) & $41(48 \%)$ & \\
\hline T3 & $6(8 \%)$ & 7 (8\%) & \\
\hline Lymph node involvement & & & .864 \\
\hline No & $37(51 \%)$ & $42(49 \%)$ & \\
\hline N1 & 27 (37\%) & $30(35 \%)$ & \\
\hline $\mathrm{N} 2 / 3$ & $9(12 \%)$ & $13(15 \%)$ & \\
\hline Estrogen receptor-positive & 58 (79\%) & 57 (67\%) & .081 \\
\hline $\begin{array}{l}\text { Progesterone } \\
\text { receptor-positive }\end{array}$ & $48(66 \%)$ & 44 (52\%) & .075 \\
\hline Triple-negative & 10 (14\%) & 19 (22\%) & .161 \\
\hline \multicolumn{4}{|l|}{ Chemotherapy agent } \\
\hline Doxorubicin & $52(71 \%)$ & $57(67 \%)$ & .571 \\
\hline Cyclophosphamide & 73 (100\%) & 85 (100\%) & - \\
\hline Docetaxel & $21(29 \%)$ & $29(34 \%)$ & .471 \\
\hline Paclitaxel & $48(66 \%)$ & $56(66 \%)$ & .986 \\
\hline Trastuzumab & $23(32 \%)$ & $22(26 \%)$ & .435 \\
\hline Radiation & 64 (88\%) & 60 (71\%) & .206 \\
\hline
\end{tabular}

Abbreviation: NExT, Nutrition and Exercise During Adjuvant Treatment.

number of cycles with neutropenia did not differ between groups $(P=.492$ and $P=.381$, respectively). The maximum neutropenia severity level experienced (ie, none, grade 1 , grade $\geq 2$ ) did not differ between groups $(P=.798$; Figure $3 \mathrm{~A})$. Almost all patients experienced 

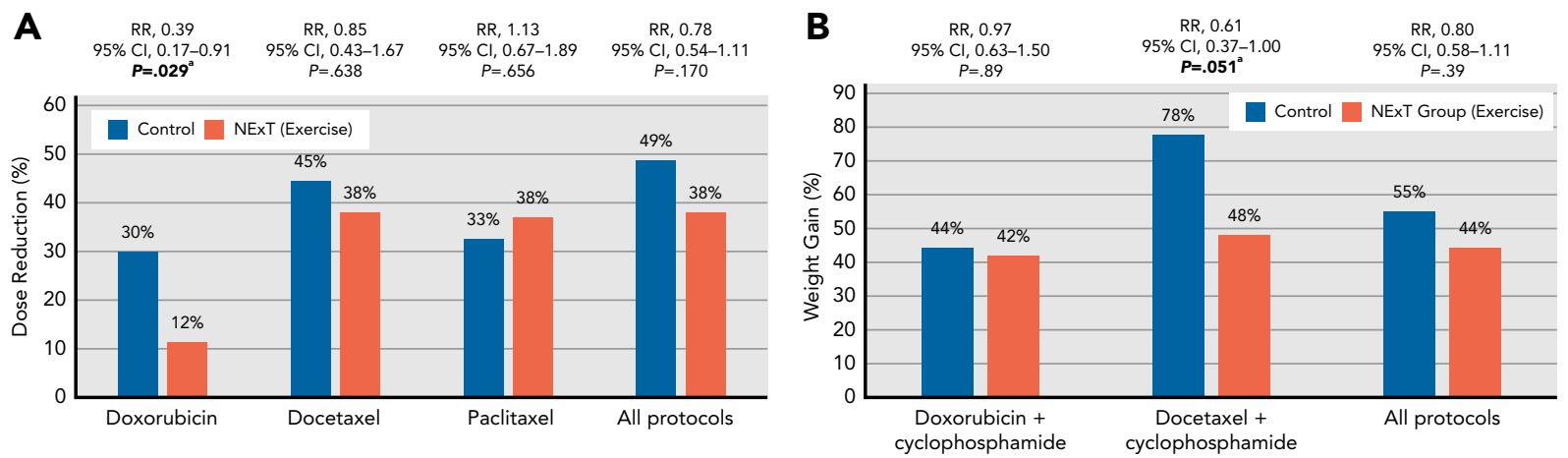

Figure 2. Agent-specific group differences in (A) chemotherapy dose reductions and (B) weight gain. Abbreviations: NExT, Nutrition and Exercise During Adjuvant Treatment; RR, relative risk.

aBold indicates statistically significant $P$ value.

anemia for at least 1 chemotherapy cycle, and this did not differ between the EX and CTR groups (95\% vs 96\%; RR, 0.98 ; 95\% CI, 0.92-1.05; $P=.580$; NNT, 54; Figure 3B). The absolute or relative (percentage of total) number of cycles with anemia did not differ between groups $(P=.149$ and $P=.087$, respectively). The maximum anemia severity level experienced (ie, none, mild, moderate, severe) did not differ between groups $(P=.768$; Figure $3 \mathrm{~B})$. The $\mathrm{RR}$ of weight gain during chemotherapy treatment did not differ between the EX and CTR groups (44\% vs 55\%, respectively; RR, 0.80; $95 \%$ CI, 0.58-1.11; $P=.176$; NNT, 9; Figure 2B).

Neuropathy was the most common reason for dose reduction in both groups and did not differ between groups (Table 2). Dose reductions due to neutropenia $(P=.027)$, other infections $(P=.049)$, and fatigue $(P=.037)$ were less common in the EX group compared with the CTR group, whereas dose reductions due to mucositis were more common ( $P=.023$; Table 2$)$. The most common reasons for dose delays in both groups were other illness and neutropenia, which did not differ between groups (Table 3).

\section{Survival Outcomes}

The extent of censorship for survival analysis was similar between the groups (DFS, 89\%; OS, 93\%). After a median follow-up of 70 months (range, 54-84 months) among surviving patients, there were 17 DFS events and 11 OS events. There was no difference between the EX and CTR groups in DFS events $(n=8$ [11\%] vs $n=9$ [11\%]; log-rank test, $P=.78$; Figure $4 \mathrm{~A})$ or OS events $(\mathrm{n}=5[7 \%]$ vs $\mathrm{n}=6$ [7\%]; log-rank test, $P=.974$; Figure $4 \mathrm{~B}$ ).

\section{Agent-Specific Effects of Exercise}

There was a significantly lower risk of a doxorubicin dose reduction in the EX group compared with the CTR group ( $12 \%$ vs $30 \%$; RR, 0.39; $95 \%$ CI, $0.17-0.91 ; P=.029$; NNT, 5; Figure 2A). With receipt of docetaxel and cyclophosphamide, there was a significantly reduced risk of weight gain in the EX group compared with the CTR group (48\% vs 78\%; RR, 0.61; 95\% CI, 0.37-1.00; $P=.051$; NNT, 3; Figure 2B).

\section{Discussion}

We found no difference in overall treatment tolerance and survival in women with early-stage breast cancer who participated in a supervised aerobic and resistance training intervention during adjuvant treatment compared with historical control participants matched for age, body mass index, diagnosis, and treatment from the same clinical site. There was a trend toward attendance of $\geq 50 \%$ of 3 weekly supervised exercise sessions during chemotherapy treatment, resulting in higher average RDI and reduced risk of a clinically relevant chemotherapy RDI reduction $(<85 \%)$; however, our study design did not allow an analysis of causal direction. Generally, poor treatment tolerance may also impact the ability of an individual to participate in an exercise intervention, confounding the ability to examine the impact of exercise adherence on treatment tolerance outcomes.

A novel finding in our study is that exercise may have chemotherapy agent-specific effects on some clinical outcomes. We found that supervised exercise programming during chemotherapy reduced the relative and absolute risks of a dose reduction in doxorubicin by $60 \%$ and $18 \%$, respectively, and there was no effect on taxane dose reductions. To our knowledge, all other studies reporting the effect of exercise on chemotherapy tolerance have combined all chemotherapy regimens ${ }^{4}$ and may have missed a potential agent-specific effect. Dose reductions are closely related to the toxicity profile of a regimen, and anthracyclines, including doxorubicin, are considered among the chemotherapy agents with the most toxic side-effect profile. ${ }^{20}$ Although the reason for this regimen-specific effect is unknown, it has been suggested that there may be greater potential for exercise to improve tolerance when tolerance is lower. ${ }^{21}$ Although highly toxic, anthracyclines are also among the most 
A

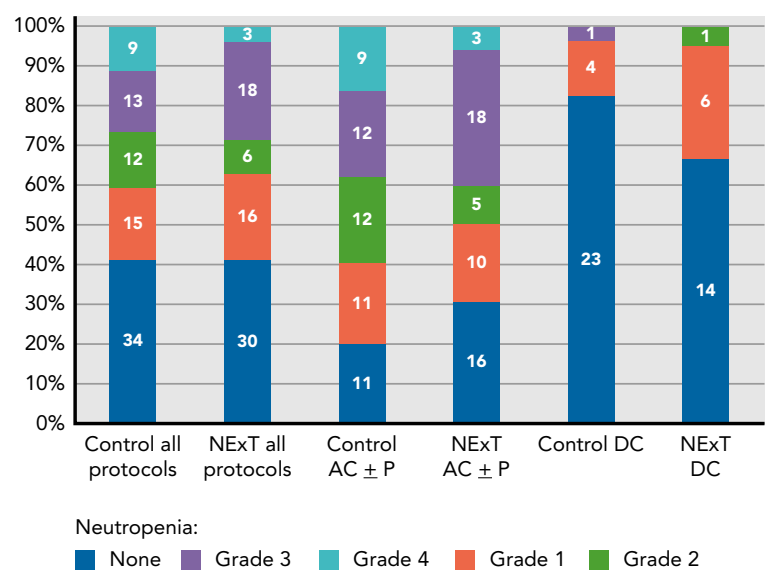

B

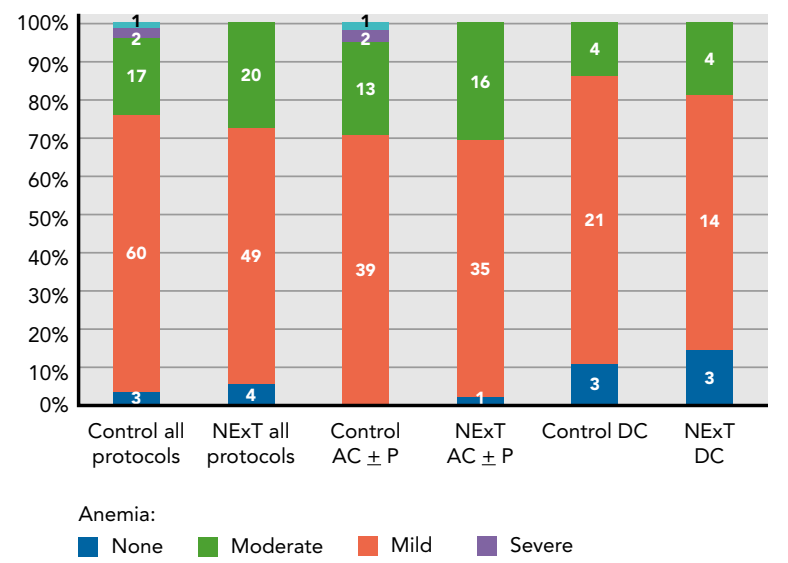

Figure 3. Absolute (count) and relative (percentage) numbers of patients by severity of (A) neutropenia and (B) anemia experienced, divided by group and chemotherapy type.

Abbreviations: $A C \pm P$, doxorubicin + cyclophosphamide with or without sequential paclitaxel; DC, docetaxel + cyclophosphamide; NExT, Nutrition and Exercise During Adjuvant Treatment.

effective for treatment of breast cancer, ${ }^{20}$ and therefore interventions to reduce the risk of dose-limiting toxicities for anthracycline-based regimens are critical.

There was also a positive effect of exercise on prevention of weight gain during docetaxel + cyclophosphamide chemotherapy treatment. The NExT supervised exercise programming reduced the relative and absolute risks of weight gain on this regimen by $38 \%$ and $30 \%$, respectively. A potential reason for this benefit could be related to the high incidence of this side effect (ie, $78 \%$ of the control group gained weight across only 4 chemotherapy cycles), which leaves more room for improvement. Postdiagnosis weight gain has been associated with breast cancer recurrence and mortality in several studies. ${ }^{22}$ Interventions such as exercise that can help to avoid weight gain during chemotherapy or improve postdiagnosis body composition may improve short- and long-term quality of

Table 2. Reasons for Dose Reductions

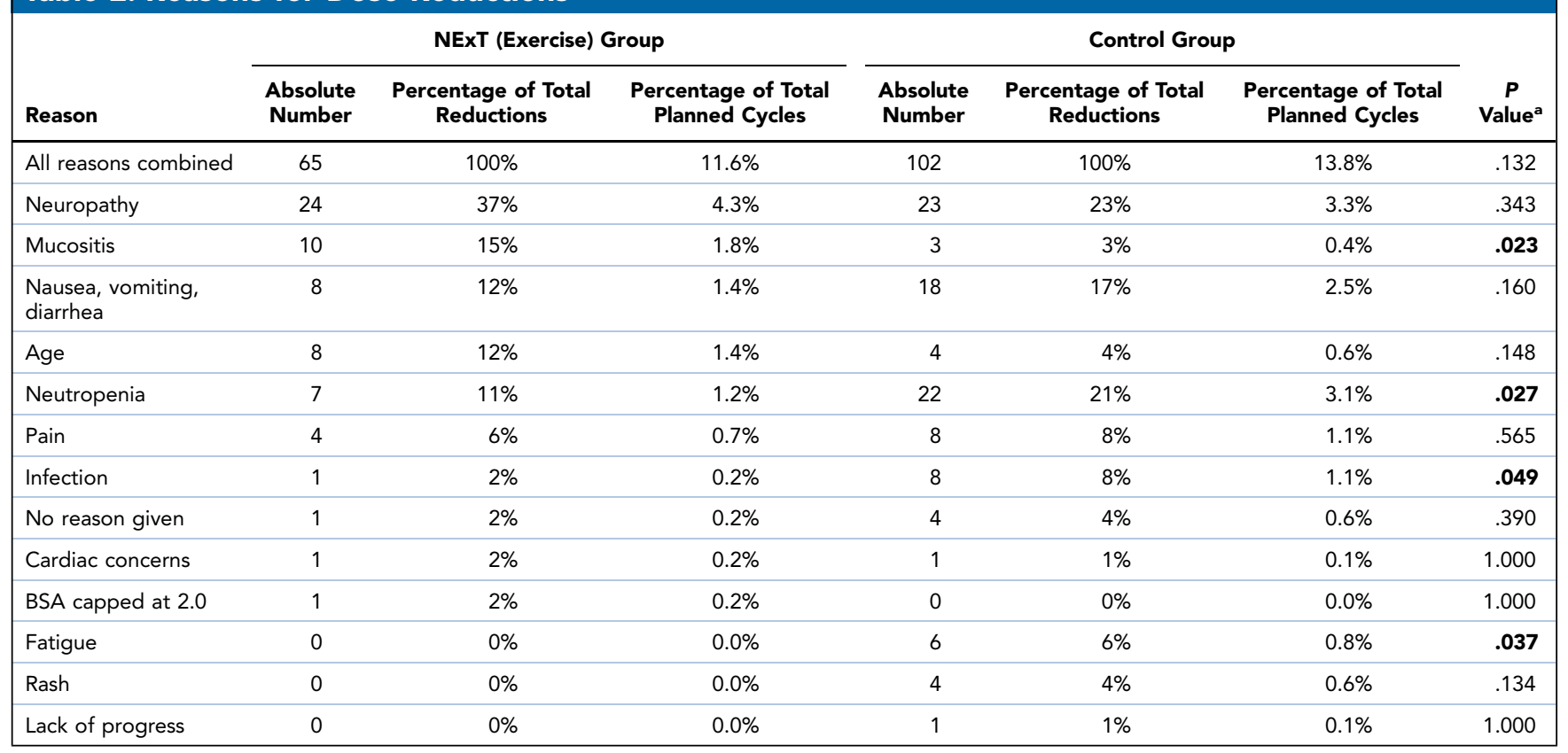

Abbreviations: BSA, body surface area; NExT, Nutrition and Exercise During Adjuvant Treatment.

aBold indicates statistically significant $P$ value. 
Table 3. Reasons for Dose Delay

\begin{tabular}{|c|c|c|c|c|c|c|c|}
\hline Reason & \multicolumn{3}{|c|}{ NExT Group (Exercise) } & \multicolumn{3}{|c|}{ Control Group } & $\begin{array}{c}P \\
\text { Value }^{a}\end{array}$ \\
\hline Other illness & 7 & $30 \%$ & $1.2 \%$ & 4 & $15 \%$ & $0.6 \%$ & .231 \\
\hline Neutropenia & 7 & $30 \%$ & $1.2 \%$ & 9 & $35 \%$ & $1.3 \%$ & .963 \\
\hline Patient request & 2 & $9 \%$ & $0.3 \%$ & 3 & $12 \%$ & $0.4 \%$ & 1.000 \\
\hline Insurance & 2 & $9 \%$ & $0.3 \%$ & 1 & $4 \%$ & $0.1 \%$ & .587 \\
\hline Mucositis & 1 & $4 \%$ & $0.2 \%$ & 1 & $4 \%$ & $0.1 \%$ & 1.000 \\
\hline Nausea & 1 & $4 \%$ & $0.2 \%$ & 1 & $4 \%$ & $0.1 \%$ & 1.000 \\
\hline Rash & 0 & $0 \%$ & $0.0 \%$ & 1 & $4 \%$ & $0.1 \%$ & 1.000 \\
\hline
\end{tabular}

Abbreviation: NExT, Nutrition and Exercise During Adjuvant Treatment.

aBold indicates statistically significant $P$ value.

life and enhance prognosis among women with breast cancer. $^{22}$

Myelosuppression is a common chemotherapy toxicity associated with considerable morbidity, mortality, and costs. ${ }^{23}$ Although the risk of developing neutropenia and anemia did not differ between groups, the likelihood of a dose reduction due to neutropenia, other (nonneutropenic) infections, or fatigue was significantly less in the EX group than in the CTR group. This suggests that participating in exercise during chemotherapy may attenuate the extent to which myelosuppression impacts treatment tolerance. This analysis also provides a real-world estimate of the prevalence of neutropenia and anemia. Approximately $60 \%$ of all patients were defined as neutropenic, and $96 \%$ experienced anemia for at

A

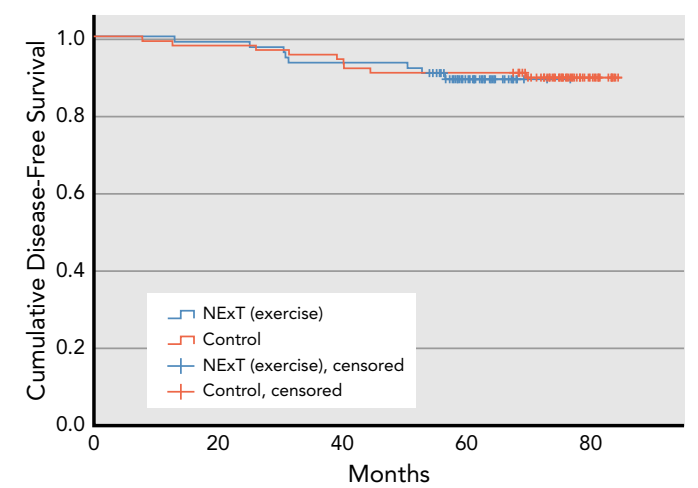

least one chemotherapy cycle. Although there are clinical thresholds for neutropenia that require dose adjustments, treatment is typically only interrupted for anemia in extreme cases (ie, severe or life-threatening anemia, which was documented in only $2 \%$ of patients in this sample) or for indirect reasons such as substantial patient-reported fatigue. However, even mild anemia can negatively affect a patient's energy levels, desire to engage in exercise, and cardiovascular responses to exercise. Exercise professionals working with individuals receiving chemotherapy should be aware of this ubiquitous side effect and adapt their exercise counseling and prescription approaches accordingly.

A strength of this analysis was the comprehensive and standardized reporting of treatment tolerance (ie, planned
B

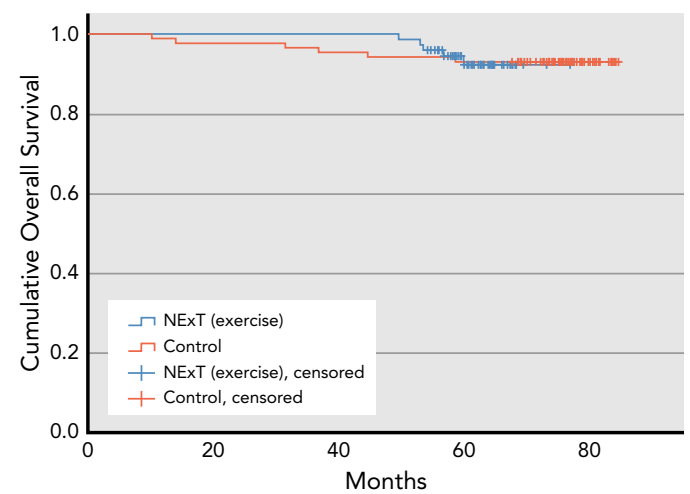

Figure 4. (A) Disease-free and (B) overall survival for NExT (exercise) and control groups.

Abbreviation: NExT, Nutrition and Exercise During Adjuvant Treatment. 
vs received dose and schedule, $\mathrm{CBC}$ counts, reasons for dose disruptions) and survival (ie, DFS and OS). Although our study design was limited by nonrandomization, our historical cohort was randomly selected and matched for age, treatment type, and location. Doses and regimens of chemotherapy prescribed did not differ between groups, and there is a high likelihood that the overall treatment approach was similar between groups, with the exception of the exercise intervention. The close historical period selected for the comparison group did not overlap with any practice-wide changes to care delivery. The relatively small sample size may have limited our power to assess our exploratory analyses of effect of exercise on survival, as well as the per-protocol and chemotherapy regimen subgroup analyses. Another limitation is that we were unable to quantify the effect of the single nutrition class on our outcomes.

\section{Conclusions}

A supervised aerobic and resistance exercise intervention provided during adjuvant chemotherapy for early-stage breast cancer did not improve treatment tolerance or efficacy compared with historical controls. However, the finding that the impact of exercise on treatment tolerance may differ by type of chemotherapy agents received warrants further investigation. Grouping all treatment types into one analysis may miss potential effects of exercise as a supportive therapy for adjuvant chemotherapy.

Submitted March 16, 2020; accepted for publication June 3, 2020.

Author contributions: Study concept: Kirkham, Gelmon Van Patten, McKenzie, Campbell. Funding acquisition: Van Patten, Campbell. Data acquisition: Kirkham, Gelmon, Van Patten, Bland, Wollmann, Landry, Campbell. Provided patients: Gelmon. Data analysis and interpretation: Kirkham. Manuscript preparation: Kirkham, Campbell.

Disclosures: The authors have disclosed that they have not received any financial consideration from any person or organization to support the preparation, analysis, results, or discussion of this article.

Funding: This work was supported by funding from the BC Cancer Agency (Campbell) and the Canadian Institutes of Health Research (Kirkham).

Correspondence: Kristin L. Campbell, PhD, University of British Columbia, 212-2177 Wesbrook Mall, Vancouver, BC, V6T 1Z3, Canada.

Email: kristin.campbell@ubc.ca

\section{References}

1. Courneya KS, Rogers LQ, Campbell KL, et al. Top 10 research questions related to physical activity and cancer survivorship. Res Q Exerc Sport 2015;86:107-116.

2. van Waart $H$, Stuiver MM, van Harten $W H$, et al. Effect of low-intensity physical activity and moderate- to high-intensity physical exercise during adjuvant chemotherapy on physical fitness, fatigue, and chemotherapy completion rates: results of the PACES randomized clinical trial. J Clin Oncol 2015;33:1918-1927.

3. Lyman $\mathrm{GH}$. Impact of chemotherapy dose intensity on cancer patient outcomes. J Natl Compr Canc Netw 2009;7:99-108.

4. Bland KA, Zadravec K, Landry T, et al. Impact of exercise on chemotherapy completion rate: a systematic review of the evidence and recommendations for future exercise oncology research. Crit Rev Oncol Hematol 2019;136:79-85

5. Courneya KS, Segal RJ, Mackey JR, et al. Effects of aerobic and resistance exercise in breast cancer patients receiving adjuvant chemotherapy: a multicenter randomized controlled trial. J Clin Oncol 2007;25:4396-4404.

6. Courneya KS, Segal RJ, McKenzie DC, et al. Effects of exercise during adjuvant chemotherapy on breast cancer outcomes. Med Sci Sports Exerc 2014;46:1744-1751.

7. Hayes SC, Steele ML, Spence RR, et al. Exercise following breast cancer: exploratory survival analyses of two randomised, controlled trials. Breast Cancer Res Treat 2018;167:505-514.

8. Courneya KS, Booth CM, Gill S, et al. The Colon Health and Life-Long Exercise Change trial: a randomized trial of the National Cancer Institute of Canada Clinical Trials Group. Curr Oncol 2008;15:279-285.

9. Newton RU, Kenfield SA, Hart NH, et al. Intense Exercise for Survival among Men with Metastatic Castrate-Resistant Prostate Cancer (INTERVAL-GAP4): a multicentre, randomised, controlled phase III study protocol. BMJ Open 2018;8:e022899.

10. Hayes S, Friedlander ML, Obermair A, et al. Exercise during Chemotherapy for Ovarian Cancer (ECHO): study design features and outcomes of a Cancer Australia and Cancer Council Australia funded randomized, controlled trial [abstract]. Int J Gynecol Cancer 2014;24: 200-201.

11. Wiskemann J, Kuehl R, Dreger P, et al. Physical Exercise Training versus Relaxation in Allogeneic stem cell transplantation (PETRA Study) - rationale and design of a randomized trial to evaluate a yearlong exercise intervention on overall survival and side-effects after allogeneic stem cell transplantation. BMC Cancer 2015;15:619.

12. Hatswell AJ, Sullivan WG. Creating historical controls using data from a previous line of treatment - two non-standard approaches. Stat Methods Med Res 2020;29:1563-1572.

13. Kirkham AA, Lloyd MG, Claydon VE, et al. A longitudinal study of the association of clinical indices of cardiovascular autonomic function with breast cancer treatment and exercise training. Oncologist 2019;24: 273-284.

14. Kirkham AA, Van Patten CL, Gelmon KA, et al. Effectiveness of oncologist-referred exercise and healthy eating programming as a part of supportive adjuvant care for early breast cancer. Oncologist 2018;23. 105-115.

15. Kirkham AA, Bonsignore A, Bland KA, et al. Exercise prescription and adherence for breast cancer: one size does not FITT all. Med Sci Sports Exerc 2018;50:177-186.

16. Kirkham AA, Bland KA, Wollmann $\mathrm{H}$, et al. Maintenance of fitness and quality-of-life benefits from supervised exercise offered as supportive care for breast cancer. J Natl Compr Canc Netw 2019;17:695-702.

17. Courneya KS, McKenzie DC, Mackey JR, et al. Effects of exercise dose and type during breast cancer chemotherapy: multicenter randomized trial. J Natl Cancer Inst 2013;105:1821-1832.

18. Weycker D, Barron R, Edelsberg J, et al. Incidence of reduced chemotherapy relative dose intensity among women with early stage breast cancer in US clinical practice. Breast Cancer Res Treat 2012;133:301-310.

19. Rosenbaum K, Wang J, Pierson RN Jr, et al. Time-dependent variation in weight and body composition in healthy adults. JPEN J Parenter Entera Nutr 2000;24:52-55.

20. Henderson IC. Can we abandon anthracyclines for early breast cancer patients? Oncology (Williston Park) 2011;25:115-124, 127

21. Kirkham AA. Supervised, multimodal exercise: the chemotherapy supportive therapy that almost does it all. Oncologist 2020;25:3-5.

22. Makari-Judson G, Braun B, Jerry DJ, et al. Weight gain following breast cancer diagnosis: implication and proposed mechanisms. World J Clin Oncol 2014;5:272-282.

23. Kuderer NM, Dale DC, Crawford J, et al. Mortality, morbidity, and cost associated with febrile neutropenia in adult cancer patients. Cancer 2006; 106:2258-2266. 